\title{
The Diagonalization Paradox
}

\author{
Ron Ragusa
}

May 2020

\begin{abstract}
In 1891 Georg Cantor published his Diagonal Argument which, he asserted, proved that the real numbers cannot be put into a one-toone correspondence with the natural numbers.In this paper we will see how by varying the initial conditions of the demonstration we can use Cantor's method to produce a one-to-one correspondence between the set of natural numbers and the set of infinite binary decimals in the open interval $(0,1)$.
\end{abstract}




\section{Introduction}

In 1891 Georg Cantor published his Diagonal Argument which, he asserted, proved that the real numbers cannot be put into a one-to-one correspondence with the natural numbers. Cantor's proof relies on the fact that a complete list of real numbers over a given interval say, $(0,1)$ cannot, in fact be realized. That is to say, the infinite set of real numbers in $(0,1)$ cannot be listed linearly in its entirety and thus is uncountable. Cantor's reasoning is that since the real number list must necessarily be incomplete then any attempt to put the numbers in $(0,1)$ in a one-to-one correspondence with the natural numbers is bound to fail. To demonstrate, we begin with a matched list of real infinite binary decimal numbers in $(0,1)$ which we assume is complete. Each number in the list is matched with a corresponding natural number. Then any real number found not to be contained in the list will have no natural number to pair with. Cantor's diagonal method produces numbers not contained in the list and from this he concludes that the set of real numbers in the interval $(0,1)$ must have more members than the set of all natural numbers.

In this paper we will see how by varying the initial conditions of the demonstration we can use Cantor's method to produce a one-to-one correspondence between the set of natural numbers and the set of infinite binary decimals in the open interval $(0,1)$. We concede that the initial list of infinite binary decimals is, in fact, incomplete and that the diagonal method does produce a number not contained in the list. Also we'll agree that there are an infinite number of binary decimal numbers in the interval that are not in the list. We are not arguing that Cantor's proof is incorrect, only that it's not the last word on the subject. We will see how using the same diagonal method we can create infinitely many binary decimal numbers contained in the interval and that for each number we so create each will correspond with one and only one natural number.

\section{Initial Conditions, Cantor's 1891 Proof}

1. The set $B$ of infinite binary decimals on the interval $(0,1)$,

$$
B=\{d: 0<d<1\}
$$

2. An assumed complete list $L$, of the elements of $B$. 


\begin{tabular}{llllllllllll}
\multicolumn{11}{c}{$\mathrm{d}$} \\
\hline. & 0 & 1 & 0 & 1 & 0 & 1 & 0 & 1 & 0 & 1 & $\ldots$ \\
. & 1 & 1 & 0 & 1 & 1 & 0 & 1 & 1 & 0 & 1 & $\ldots$ \\
. & 0 & 0 & 1 & 0 & 0 & 1 & 0 & 0 & 1 & 0 & $\ldots$ \\
. & 0 & 0 & 0 & 1 & 0 & 0 & 0 & 1 & 0 & 0 & $\ldots$ \\
. & 1 & 0 & 1 & 0 & 1 & 0 & 1 & 0 & 1 & 0 & $\ldots$
\end{tabular}

3. The set of natural numbers $\mathbb{N}$,

$$
\mathbb{N}=\{1,2,3, \ldots\}
$$

4. The following arrangement matching each item in $\mathrm{L}$ with an element of $\mathbb{N}$ :

\begin{tabular}{|c|c|c|c|c|c|c|c|c|c|c|c|}
\hline$n$ & & & & & $\mathrm{~d}$ & & & & & & \\
\hline 1 & .0 & 1 & 0 & 1 & 0 & 1 & 0 & 1 & 0 & 1 & \\
\hline 2 & 1 & 1 & 0 & 1 & 1 & 0 & 1 & 1 & 0 & 1 & .. \\
\hline 3 & 0 & 0 & 1 & 0 & 0 & 1 & 0 & 0 & 1 & 0 & $\ldots$ \\
\hline 4 & 0 & 0 & 0 & 1 & 0 & 0 & 0 & 1 & 0 & 0 & $\ldots$ \\
\hline 5 & 1 & 1 & 1 & 0 & 1 & 1 & 1 & 0 & 1 & 1 & . \\
\hline 6 & 0 & 0 & 0 & 0 & 1 & 0 & 0 & 0 & 0 & 1 & . \\
\hline 7 & 1 & 1 & 1 & 1 & 0 & 1 & 1 & 1 & 1 & 0 & $\ldots$ \\
\hline 8 & 0 & 0 & 1 & 1 & 0 & 0 & 1 & 1 & 0 & 0 & . \\
\hline 9 & 1 & 1 & 0 & 0 & 1 & 1 & 0 & 0 & 1 & 1 & $\ldots$ \\
\hline 10 & 1 & 0 & 1 & 0 & 1 & 0 & 1 & 0 & 1 & 0 & \\
\hline
\end{tabular}

\section{Creating a number $Y$ not contained in $L$}

Next we will use the diagonal argument to construct a binary decimal number that is not in the list by flipping the first digit of the number in the first row, the second digit of the number in the second row, the third digit in the number in the third row and so on. The resulting table will appear as follows: 


\begin{tabular}{c|cccccccccccc}
$\mathrm{n}$ & & & & & & $\mathrm{d}$ & & & & & & \\
\hline 1 &. & 0 & 1 & 0 & 1 & 0 & 1 & 0 & 1 & 0 & 1 & $\ldots$ \\
2 &. & 1 & 1 & 0 & 1 & 1 & 0 & 1 & 1 & 0 & 1 & $\ldots$ \\
3 &. & 0 & 0 & 1 & 0 & 0 & 1 & 0 & 0 & 1 & 0 & $\ldots$ \\
4 &. & 0 & 0 & 0 & 1 & 0 & 0 & 0 & 1 & 0 & 0 & $\ldots$ \\
5 &. & 1 & 1 & 1 & 0 & 1 & 1 & 1 & 0 & 1 & 1 & $\ldots$ \\
6 &. & 0 & 0 & 0 & 0 & 1 & 0 & 0 & 0 & 0 & 1 & $\ldots$ \\
7 &. & 1 & 1 & 1 & 1 & 0 & 1 & 1 & 1 & 1 & 0 & $\ldots$ \\
8 &. & 0 & 0 & 1 & 1 & 0 & 0 & 1 & 1 & 0 & 0 & $\ldots$ \\
9 &. & 1 & 1 & 0 & 0 & 1 & 1 & 0 & 0 & 1 & 1 & $\ldots$ \\
10 &. & 1 & 0 & 1 & 0 & 1 & 0 & 1 & 0 & 1 & 0 & $\ldots$ \\
$\ldots$ & & & & & & $\ldots$ & & & & & & \\
\hline $\mathrm{Y}$ &. & $\mathbf{1}$ & $\mathbf{0}$ & $\mathbf{0}$ & $\mathbf{0}$ & $\mathbf{0}$ & $\mathbf{1}$ & $\mathbf{0}$ & $\mathbf{0}$ & $\mathbf{0}$ & $\mathbf{1}$ & $\ldots$ \\
& & & & & & & & & & & &
\end{tabular}

The number $Y$ will differ at each $n^{\text {th }}$ digit from the number in the $n^{\text {th }}$ row of the table. Therefore $Y$ cannot be in $L$ and cannot be matched with a natural number. Since $Y$ cannot be paired with a natural number not already in $L$, Cantor reasoned that the set of numbers in $(0,1)$ must be larger than the set of natural numbers.

\section{Changing the Initial Conditions}

We will construct a proof with slightly different initial conditions that will demonstrate that the real and natural numbers can be arranged in such a way as to match one natural number with one real number in the interval without exception. Our new initial conditions are as follows:

1. The set $\mathbb{N}$ of natural numbers,

$$
\mathbb{N}=\{1,2,3, \ldots\}
$$

2. The set $\mathbb{N}_{e}$ of even natural numbers,

$$
\mathbb{N}_{e}=\{2,4,6, \ldots\}
$$

3. The set $\mathbb{N}_{o}$ of odd natural numbers,

$$
\mathbb{N}_{o}=\{1,3,5, \ldots\}
$$

4. From set theory we know that:

$$
f: \mathbb{N} \mapsto \mathbb{N}_{o}, f: \mathbb{N} \mapsto \mathbb{N}_{e} \text { and } \mathbb{N}_{o} \cup \mathbb{N}_{e}=\mathbb{N} \text { therefore } f: \mathbb{N} \mapsto \mathbb{N}_{o} \cup \mathbb{N}_{e}
$$


5. The set $B$ of infinite binary decimals in the interval $(0,1)$,

$$
B=\{d: 0<d<1\}
$$

6. An assumed to be complete list $L$, of the elements of $B$.

\begin{tabular}{cccccccccccc}
\multicolumn{10}{c}{$\mathrm{d}$} \\
\hline. & 0 & 1 & 0 & 1 & 0 & 1 & 0 & 1 & 0 & 1 & $\ldots$ \\
. & 1 & 1 & 0 & 1 & 1 & 0 & 1 & 1 & 0 & 1 & $\ldots$ \\
. & 0 & 0 & 1 & 0 & 0 & 1 & 0 & 0 & 1 & 0 & $\ldots$ \\
. & 0 & 0 & 0 & 1 & 0 & 0 & 0 & 1 & 0 & 0 & $\ldots$ \\
. & 1 & 0 & 1 & 0 & 1 & 0 & 1 & 0 & 1 & 0 & $\ldots$ \\
& & & & & & $\ldots$ & & & & &
\end{tabular}

We now begin as Cantor did, by constructing a number $Y$ not in $L$. Our list is constructed slightly differently than Cantor's in that we are matching each real number in $L$ with an element of $\mathbb{N}_{o}$ as shown below:

\begin{tabular}{c|cccccccccccc}
$\mathrm{n}$ & \multicolumn{10}{c}{$\mathrm{d}$} & & \\
\hline 1 & $\cdot$ & 0 & 1 & 0 & 1 & 0 & 1 & 0 & 1 & 0 & 1 & $\ldots$ \\
3 & $\cdot$ & 1 & 1 & 0 & 1 & 1 & 0 & 1 & 1 & 0 & 1 & $\ldots$ \\
5 & $\cdot$ & 0 & 0 & 1 & 0 & 0 & 1 & 0 & 0 & 1 & 0 & $\ldots$ \\
7 & $\cdot$ & 0 & 0 & 0 & 1 & 0 & 0 & 0 & 1 & 0 & 0 & $\ldots$ \\
9 & $\cdot$ & 1 & 1 & 1 & 0 & 1 & 1 & 1 & 0 & 1 & 1 & $\ldots$ \\
11 &. & 0 & 0 & 0 & 0 & 1 & 0 & 0 & 0 & 0 & 1 & $\ldots$ \\
13 & $\cdot$ & 1 & 1 & 1 & 1 & 0 & 1 & 1 & 1 & 1 & 0 & $\ldots$ \\
15 & $\cdot$ & 0 & 0 & 1 & 1 & 0 & 0 & 1 & 1 & 0 & 0 & $\ldots$ \\
17 & $\cdot$ & 1 & 1 & 0 & 0 & 1 & 1 & 0 & 0 & 1 & 1 & $\ldots$ \\
19 & $\cdot$ & 1 & 0 & 1 & 0 & 1 & 0 & 1 & 0 & 1 & 0 & $\ldots$ \\
$\ldots$ & & & & & $\ldots$ & & & & & & \\
\hline $\mathrm{Y}$ & $\cdot$ & $\mathbf{1}$ & $\mathbf{0}$ & $\mathbf{0}$ & $\mathbf{0}$ & $\mathbf{0}$ & $\mathbf{1}$ & $\mathbf{0}$ & $\mathbf{0}$ & $\mathbf{0}$ & $\mathbf{1}$ & $\ldots$
\end{tabular}

As before, $Y$ is not in $L$. The next step requires that we enter $Y$ into $L$. $L$ has no end so we put $Y$ in at the beginning of $L$ and match it with the first element of the set $\mathbb{N}_{e}$, which is 2 . See below. 


\begin{tabular}{c|cccccccccccc}
$\mathrm{n}$ & & & & & & $\mathrm{d}$ & & & & & & \\
\hline 2 &. & 1 & 0 & 0 & 0 & 0 & 1 & 0 & 0 & 0 & 1 & $\ldots$ \\
1 &. & 0 & 1 & 0 & 1 & 0 & 1 & 0 & 1 & 0 & 1 & $\ldots$ \\
3 &. & 1 & 1 & 0 & 1 & 1 & 0 & 1 & 1 & 0 & 1 & $\ldots$ \\
5 &. & 0 & 0 & 1 & 0 & 0 & 1 & 0 & 0 & 1 & 0 & $\ldots$ \\
7 &. & 0 & 0 & 0 & 1 & 0 & 0 & 0 & 1 & 0 & 0 & $\ldots$ \\
9 &. & 1 & 1 & 1 & 0 & 1 & 1 & 1 & 0 & 1 & 1 & $\ldots$ \\
11 &. & 0 & 0 & 0 & 0 & 1 & 0 & 0 & 0 & 0 & 1 & $\ldots$ \\
13 &. & 1 & 1 & 1 & 1 & 0 & 1 & 1 & 1 & 1 & 0 & $\ldots$ \\
15 &. & 0 & 0 & 1 & 1 & 0 & 0 & 1 & 1 & 0 & 0 & $\ldots$ \\
17 &. & 1 & 1 & 0 & 0 & 1 & 1 & 0 & 0 & 1 & 1 & $\ldots$ \\
$\ldots$ & & & & & & $\ldots$ & & & & & & \\
\hline $\mathrm{Y}$ &. & $\mathbf{0}$ & $\mathbf{0}$ & $\mathbf{1}$ & $\mathbf{1}$ & $\mathbf{1}$ & $\mathbf{0}$ & $\mathbf{1}$ & $\mathbf{0}$ & $\mathbf{1}$ & $\mathbf{0}$ & $\ldots$
\end{tabular}

We will repeat the procedure with the new number added to the list to create another number $Y$ also not in the list and match this number with the next element of $\mathbb{N}_{e}$ and add it to the list. Our list now looks like:

\begin{tabular}{c|cccccccccccc}
$\mathrm{n}$ & \multicolumn{10}{|c}{$\mathrm{d}$} & & \\
\hline 4 &. & 0 & 0 & 1 & 1 & 1 & 0 & 1 & 0 & 1 & 0 & $\ldots$ \\
2 &. & 1 & 0 & 0 & 0 & 0 & 1 & 0 & 0 & 0 & 1 & $\ldots$ \\
1 &. & 0 & 1 & 0 & 1 & 0 & 1 & 0 & 1 & 0 & 1 & $\ldots$ \\
3 &. & 1 & 1 & 0 & 1 & 1 & 0 & 1 & 1 & 0 & 1 & $\ldots$ \\
5 &. & 0 & 0 & 1 & 0 & 0 & 1 & 0 & 0 & 1 & 0 & $\ldots$ \\
7 &. & 0 & 0 & 0 & 1 & 0 & 0 & 0 & 1 & 0 & 0 & $\ldots$ \\
9 &. & 1 & 1 & 1 & 0 & 1 & 1 & 1 & 0 & 1 & 1 & $\ldots$ \\
11 &. & 0 & 0 & 0 & 0 & 1 & 0 & 0 & 0 & 0 & 1 & $\ldots$ \\
13 &. & 1 & 1 & 1 & 1 & 0 & 1 & 1 & 1 & 1 & 0 & $\ldots$ \\
15 &. & 0 & 0 & 1 & 1 & 0 & 0 & 1 & 1 & 0 & 0 & $\ldots$ \\
$\ldots$ & & & & & & $\ldots$ & & & & & & \\
\hline $\mathrm{Y}$ &. & $\mathbf{1}$ & $\mathbf{1}$ & $\mathbf{1}$ & $\mathbf{0}$ & $\mathbf{1}$ & $\mathbf{1}$ & $\mathbf{0}$ & $\mathbf{1}$ & $\mathbf{0}$ & $\mathbf{1}$ & $\ldots$
\end{tabular}

This procedure can be repeated over and over indefinitely expanding $L$ with real numbers from the interval not initially in $L$. After $n$ iterations our list looks like: 


\begin{tabular}{|c|c|c|c|c|c|c|c|c|c|c|c|}
\hline $\mathrm{n}$ & & & & & d & & & & & & \\
\hline $\mathrm{n}$ & & & & & $\mathrm{Y}$ & & & & & & \\
\hline 4 & 0 & 0 & 1 & 1 & 1 & 0 & 1 & 0 & 1 & 0 & \\
\hline 2 & 1 & 0 & 0 & 0 & 0 & 1 & 0 & 0 & 0 & 1 & \\
\hline 1 & 0 & 1 & 0 & 1 & 0 & 1 & 0 & 1 & 0 & 1 & \\
\hline 3 & 1 & 1 & 0 & 1 & 1 & 0 & 1 & 1 & 0 & 1 & . \\
\hline 5 & 0 & 0 & 1 & 0 & 0 & 1 & 0 & 0 & 1 & 0 & $\cdots$ \\
\hline 7 & 0 & 0 & 0 & 1 & 0 & 0 & 0 & 1 & 0 & 0 & $\ldots$ \\
\hline 9 & 1 & 1 & 1 & 0 & 1 & 1 & 1 & 0 & 1 & 1 & $\ldots$ \\
\hline 11 & 0 & 0 & 0 & 0 & 1 & 0 & 0 & 0 & 0 & 1 & . \\
\hline 13 & 1 & 1 & 1 & 1 & 0 & 1 & 1 & 1 & 1 & 0 & $\ldots$ \\
\hline 15 & 0 & 0 & 1 & 1 & 0 & 0 & 1 & 1 & 0 & 0 & $\ldots$ \\
\hline
\end{tabular}

We now have a situation where every element of $L$, at any moment in time, is matched with one and only one natural number. And going forward, no matter how many numbers $Y$ are added to the list, each will be matched with one and only one natural number.

\section{Proof that $f: \mathbb{N} \mapsto B$}

Let $B_{1}$ be defined as a subset of $B$ such that every element of $B_{1}$ is a member of $L$ before any numbers $Y$ have been created and inserted into $L$,

$$
B_{1} \subset B \text { and } B_{1}=\{d: d \in L\} .
$$

Let $B_{2}$ be defined as a subset of $B$ such that every element of $B_{2}$ is not a member of $L$ before any numbers $Y$ have been created and inserted into $L$,

$$
B_{2} \subset B \text { and } B_{2}=\{d: d \notin L\} .
$$

The union of $B_{1}$ and $B_{2}$ is the set $B$,

$$
B_{1} \cup B_{2}=B .
$$

For every $d \in B_{1}$ we have one and only one corresponding $n \in \mathbb{N}_{o}$ therefore

$$
f: \mathbb{N}_{o} \mapsto B_{1} \text { exists. }
$$

For every $d \in B_{2}$ we have one and only one corresponding $n \in \mathbb{N}_{e}$ therefore

$$
f: \mathbb{N}_{e} \mapsto B_{2} \text { exists. }
$$


It follows that

$$
\begin{gathered}
f:\left(\mathbb{N}_{o} \cup \mathbb{N}_{e}\right) \mapsto\left(B_{1} \cup B_{2}\right) \text { exists and since } \\
\left(\mathbb{N}_{o} \cup \mathbb{N}_{e}\right)=\mathbb{N} \text { and }\left(B_{1} \cup B_{2}\right)=B \text { then } \\
f: \mathbb{N} \mapsto B
\end{gathered}
$$

This ends the demonstration and completes the proof. 